\title{
RETRATAMIENTOS EN CARA INTERNA DEL COLGAJO CORNEAL EN LASIK. ESTUDIO COMPARATIVO CON UN GRUPO CONTROL
}

\section{UNDERSURFACE ABLATION OF THE CORNEAL FLAP FOR LASIK ENHANCEMENT}

\author{
FERNÁNDEZ-VIGO J ${ }^{1}$, MACARRO A ${ }^{1}$, FERNÁNDEZ SABUGAL J²
}

\section{RESUMEN}

Objetivo: Estudiar la eficacia y seguridad de los retratamientos en cirugía LASIK comparando el tratamiento sobre el lecho estromal con el tratamiento sobre la cara interna del colgajo corneal.

Métodos: Pacientes intervenidos mediante LASIK con defecto residual negativo sometidos a retratamiento divididos en dos grupos. Grupo control: 14 ojos tratados sobre lecho estromal. Grupo estudio: 14 ojos tratados sobre cara interna del colgajo. En el grupo estudio se incluyeron aquellos pacientes en los que se preveía que el grosor del lecho post-retratamiento sería inferior a 300 micras. Se evaluaron la refracción, la agudeza visual y la córnea pre y postretratamiento a los 3 meses del retratamiento.

Resultados: Antes del retratamiento no había diferencias entre ambos grupos en lo referido a edad, sexo, paquimetría, curvatura corneal y defecto de refracción. La ablación fue de 21.5 DE 11 en el grupo estudio y 22,6 DE 7 micras en el control $(\mathrm{p}=0,796)$. La refracción post-retratamiento fue significativamente menor en ambos grupos que antes $(\mathrm{p}<0,01)$. No hubo diferencias entre el grupo control y el estudio. La agudeza visual final mejoró con respecto a la que presentaban antes del retratamien-

\section{ABSTRACT}

Objective: To study the efficacy and safety of retreatment in LASIK surgery, comparing under surface ablation of the corneal flap and stromal bed ablation techniques.

Methods: The patients studied were those who had LASIK enhancement because of a residual negative refractive error, and were divided in two groups: a Control group of 14 eyes that had ablation of the residual stromal bed and a Study group of 14 eyes that had ablation of the undersurface of the flap. In the Study group we included patients in whom we calculated that the post-enhancement treatment of the residual stromal bed would be less than 300 microns. We compared the refraction, visual acuity and cornea pre and post-retreatment, three months later.

Results: Prior to retreatment, both groups were similar and no statistically significant differences were found in age, sex, pachymetry, corneal curvature or residual refraction. Ablation was 21.5 (SD 11) microns in study group and 22.6 (SD 7) microns in control group ( $\mathrm{p}=0.796)$. Post-retreatment refraction was statistically lower in both groups than before the procedure $(\mathrm{p}<0.01)$, however there were no differences between the control and study groups. Visual

\footnotetext{
Recibido: 31/5/06. Aceptado: 20/9/07.

Universidad de Extremadura. Facultad de Medicina. Área de Oftalmología. Badajoz. España.

1 Doctor en Medicina. Universidad de Extremadura y Centro Internacional de Oftalmología Avanzada. Madrid y Badajoz.

2 Doctor en Medicina. Universidad de Extremadura.

Comunicación presentada parcialmente en el LXXI Congreso de la S.E.O. (Barcelona 2001).
}

Correspondencia:

José Fernández-Vigo

Área de Oftalmología. Facultad de Medicina

Avda. de Elvas, s/n

06011 Badajoz

España

E-mail: catoftal@unex.es 
to en ambos grupos $(p<0,01)$. No hubo diferencias entre ambos grupos. Ningún paciente perdió dos líneas de agudeza visual. Se observaron leves alteraciones en el colgajo corneal en ambos grupos.

Conclusiones: En nuestro estudio la técnica de retratamiento sobre la cara interna del colgajo es similar en eficacia y seguridad a la técnica convencional sobre el lecho estromal en defectos negativos con poco defecto residual.

Palabras clave: LASIK, cirugía refractiva, laser excimer, córnea, complicaciones.

\section{INTRODUCCIÓN}

Aunque la cirugía refractiva con láser excimer es una técnica de gran precisión para eliminar los defectos de refracción no está exenta de pequeñas incidencias como la necesidad de hacer retratamientos para ajustar los resultados. Las variaciones en las condiciones ambientales, las fluctuaciones de la energía del láser y la variabilidad inter e intraindividual hacen que los retratamientos en cirugía LASIK sean necesarios en porcentajes que varían habitualmente entre el 10 y el $20 \%$ dependiendo de diversas circunstancias (1-4). En el concepto actual la necesidad del retratamientos es vista por los pacientes como un pequeño fracaso. Por ello el retratamiento, además de garantizarnos ajustar definitivamente la refracción del paciente, debe ser muy seguro. Sin embargo desde hace tiempo se viene describiendo un incremento en el número de ectasias post-retratamiento $(5,6)$.

Maldonado (7) describió una técnica para disminuir el riesgo de ectasias haciendo los retratamientos en la cara interna del disco para así preservar un mayor grosor del lecho estromal residual demostrando unos excelentes resultados en un grupo de pacientes.

En este trabajo presentamos los resultados de un estudio comparando un grupo de pacientes retratados con la técnica convencional y un grupo retratado siguiendo la técnica de Maldonado.

\section{SUJETOS, MATERIAL Y MÉTODO}

Se ha realizado un estudio prospectivo comparativo sobre un total de 28 ojos correspondientes a 28 acuity improved after retreatment in both groups $(\mathrm{p}<0.01)$ but no statistically significant differences were found between control and study groups. No patient lost two lines of visual acuity. We observed slight alterations in the corneal flap in both groups.

Conclusions: In our study, the undersurface retreatment technique produced similar results to those achieved using conventional stromal bed techniques with both being effective and safe in patients with a small residual negative refractive error (Arch Soc Esp Oftalmol 2007; 82: 697-704).

Key words: LASIK, refractive surgery, excimer laser, cornea, complications.

pacientes divididos en dos grupos: Grupo control, en el que el retratamiento se realizaba en el lecho estromal, compuesto por 14 ojos consecutivos de 14 pacientes, en este grupo se incluyeron los ojos en los que se calculó que tras el retratamiento el grosor residual del lecho sería igual o mayor a 300 micras. Grupo estudio, en el que el retratamiento se realizaba en la cara interna del colgajo corneal, compuesto por 14 ojos consecutivos de 14 pacientes, se incluyeron aquellos ojos en los que se calculó que tras el retratamiento el grosor residual del lecho sería menor de 300 micras. Además todos debían cumplir los siguientes criterios: el grosor post-retratamiento de la córnea debe ser superior a 400 micras y el del lecho superior a 300 micras y el del colgajo superior a 100 micras. Todos los ojos habían sido intervenidos previamente mediante técnica LASIK por presentar miopía y/o astigmatismo miópico al menos tres meses antes y presentaban actualmente un defecto refractivo negativo comprobado tras cicloplejia. Se excluyeron aquellos pacientes que no cumplieran los requisitos descritos en párrafos anteriores, o que padecieran otras enfermedades oculares y sistémicas que contraindiquen la intervención. Todos los pacientes fueron informados sobre la técnica a utilizar advirtiéndoles de la posibilidad de realizar el tratamiento en cara interna previamente a la firma individualizada del consentimiento informado.

\section{Técnica quirúrgica}

La primera cirugía y el retratamiento fueron realizados por el mismo cirujano. La técnica LASIK 
aplicada en la primera cirugía ha sido la convencional, incluyendo paquimetría del lecho (sonogage corneo-gage plus ${ }^{\mathrm{TM}}$, Sonogage Inc, Cleveland, Ohio, USA), utilizando un microqueratomo LSK One de Moria con cabezal de 100 micras (Microtech, Inc., Moria, France) y aplicando su nomograma para obtener discos de aproximadamente 9,5 mm con bisagra nasal y ablación con láser excimer. El retratamiento se inicia en todos los casos mediante levantamiento del colgajo corneal y flaprhexis (8). Posteriormente se coloca el colgajo evertido sobre la punta de una hemosteta húmeda de aproximadamente $6 \mathrm{~mm}$ que se coloca sobre la conjuntiva nasal con el vértice alineado con la bisagra y la base sobre el canto interno procurando mantener su concavidad (a diferencia de la técnica convencional en la que el colgajo reposa sobre la conjuntiva nasal) (fig. 1). Se repite la paquimetría. Si el grosor del lecho estromal era suficiente para ser mayor o igual a 300 micras tras el retratamiento, el láser se aplicaba sobre el lecho. En caso de que el grosor del lecho una vez restada la ablación del retratamiento resultara inferior a 300 micras el láser se aplicaría sobre la cara interna del disco. Esta maniobra se realiza siguiendo los criterios descritos por Maldonado (7). A diferencia de su técnica y dado que el colgajo reposa sobre la hemosteta no es necesario ni estirar el colgajo, ni pedir al paciente que desplace el globo hacia el lado temporal para exponer el disco, ya que queda paralelo y aproximadamente a la altura del lecho (fig. 1). Sobre la

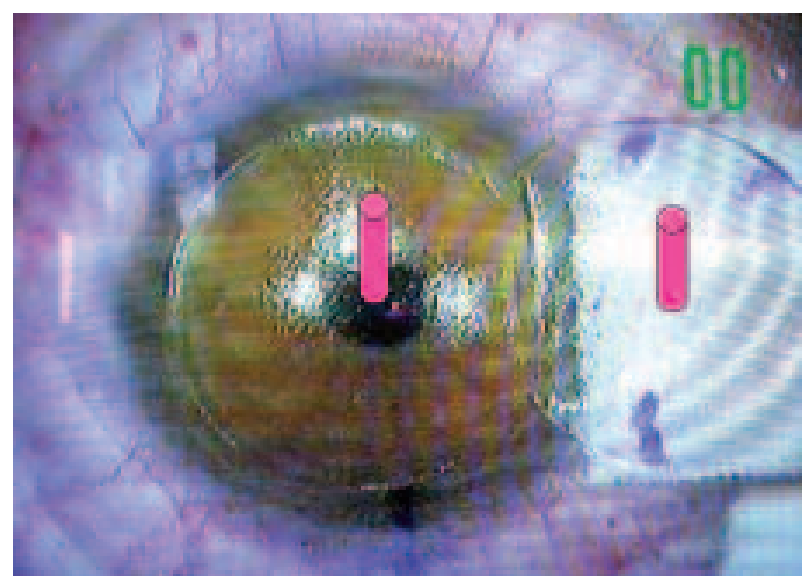

Fig. 1: Detalle de la cirugía: mostramos en el montaje fotográfico el tratamiento realizado sobre lecho o cara interna del colgajo corneal que reposa evertido sobre una hemosteta. Se aprecia la colocación, centrado y paralelismo comparado con el lecho estromal. marca realizada con violeta de genciana en epitelio y tras desplazar ligeramente la camilla se realizó el tratamiento indicando al paciente que debía seguir mirando de frente, fijando el globo mediante una pinza aplicada en conjuntiva temporal superior. Con la otra mano se protege la charnela con una hemosteta situando ambas manos paralelas y muy próximas entre sí.

Para la ablación se utilizó una zona óptica entre 5,8 y $6 \mathrm{~mm}$. con una transición de $1 \mathrm{~mm}$.

Una vez completado el tratamiento se procede como en el procedimiento primario siguiendo exactamente las mismas pautas.

\section{Parámetros de retratamiento}

Se programa el láser con los mismos criterios que en el LASIK primario. Debe considerarse como indica Maldonado que el tratamiento en la cara interna del colgajo hace el efecto de un espejo (7). Debido a que las ablaciones esféricas son simétricas no necesitan transformaciones. Sin embargo la fórmula Beta: 180- alfa es necesaria para calcular nuevas orientaciones en el eje de los tratamientos para ablaciones tóricas en el estroma del colgajo. La aplicación de esta fórmula no es precisa para los ejes de 90 y 180 grados, pero sí lo es para todos los otros ejes.

\section{Parámetros valorados}

Los pacientes fueron controlados a los 30 minutos, 24 horas, una semana, un mes y 3 meses postcirugía. Los optometristas y oftalmólogos que valoraron el post-operatorio desconocían la técnica aplicada en cada ojo. La exploración consistió en medida de la agudeza visual (AV) de lejos y cerca con y sin corrección, auto-refractometría y biomicroscopía, topografía y paquimetría. En la revisión del tercer mes se graduó tras dilatación con cicloplejia.

En la biomicroscopía se analizaron: desplazamientos del colgajo, formación de pliegues, opacificación de la córnea, estrías y epitelización de la interfase.

\section{Análisis estadístico}

Las variables cuantitativas la se expresaron en con media, rango y desviación estándar y las cuali- 
tativas con distribución de frecuencias. Se calculó la mediana de la distribución en las variables con distribuciones asimétricas. Las características de ambos grupos se compararon con el test de la t de Student para muestras independientes en variables cuantitativas y en las cualitativas con el test de la $\chi^{2}$ o prueba exacta de Fisher. El análisis comparativo de parámetros pre y postoperatorios se realizó con la t de Student para muestras apareadas. Los datos fueron recogidos en una base de datos Access (Microsoft para Windows, Microsoft Co, Portland, USA) y posteriormente exportados en Excel (Microsoft para Windows, Microsoft Co, Portland, USA) para su análisis (9).

\section{RESULTADOS}

Los resultados los referimos a la revisión del 3 mes. La población se describe en la tabla I y en las tablas II y III se realiza una descripición invidualizada de los resultados.

1. Refracción: antes del retratamiento el equivalente esférico fue de $-1,58 \mathrm{DE} 0,69$ en el grupo estudio y $-1,62$ DE 0,61 en el grupo control $(\mathrm{p}=0,853)$. Después del retratamiento la refracción medida valorando el equivalente esférico fue de $+0,08$ DE 0,02 en el grupo control (rango +0,75 a $-0,5)$ y $+0,06$ DE 0,03 (rango $+0,5$ a $-0,25$ ) en el grupo estudio $(p=0,814)$. En ambos grupos la refracción post-retratamiento es significativamente más baja que antes $(\mathrm{p}<0,01)$. En el grupo control persistió un defecto residual en tres ojos y en el grupo estudio en dos ojos (rango: - -0,25 a -0,5 en ambos grupos).

2. Agudeza visual: antes del retratamiento la AV manifiesta era de $0,58 \mathrm{DE} 0,14$ y la corregida de 0,92 DE 0,09 en el grupo control y 0,57 DE 0,14 y la AV corregida de 0,92 DE 0,1 en el grupo estudio ( $p=0,815$ y 0,876$)$. La AV manifiesta después del retratamiento fue en el grupo control de $0,9 \mathrm{DE}$ 0,11 y en el grupo estudio $0,9 \mathrm{DE} 0,1(\mathrm{p}=0,876)$. La
AV corregida fue $0,93 \mathrm{DE} 0,08$ en el grupo control y $0,91 \mathrm{DE} 0,1$ en el grupo estudio $(\mathrm{p}=0,729)$.

En el grupo estudio: la AV manifiesta post retratamiento era significativamente mayor que la pre $(p<0,01)$. No había diferencias entre la agudeza visual pre y postretratamiento corregidas ni entre la agudeza visual corregida antes del retratamiento y la espontánea post $(\mathrm{p}=0,08)$. En el grupo control: la AV manifiesta post-retratamiento era significativamente mayor que la pre $(\mathrm{p}<0,01)$. La AV pre y postretratamiento corregidas eran iguales, y no había diferencias entre la agudeza visual corregida antes del retratamiento y la manifiesta post $(\mathrm{p}=0,18)$.

En el grupo control la AV manifiesta final fue igual a la pretratamiento corregida en 10 de los 14 ojos, siendo una línea inferior en dos casos y mejor en otros dos casos. En el grupo estudio fueron 11 ojos con similar agudeza, dos ojos una línea inferior y otro ojo una línea mejor. No hubo ningún caso de pérdida de la mejor agudeza visual corregida.

3. Paquimetría: la ablación necesaria para los retratamientos fue de $21,57 \mathrm{DE} 10,86$ micras en el grupo estudio y de 22,67 DE 7,42 micras en el grupo control $(\mathrm{p}=0,796)$.

4. Biomicroscopía del segmento anterior: en ninguno de los dos grupos se observaron alteraciones significativas ya que no hubo desplazamientos del colgajo ni formación de pliegues. No se observaron signos de opacificación corneal. En dos ojos de cada grupo existían finas estrías antes del retratamiento. En el postoperatorio aparecieron en dos del grupo control (uno nuevo, otro desapareció y en otro se mantuvo) y tres en el grupo estudio (dos nuevas, desapareció en uno y en otro se mantuvo). Se apreció leve epitelización periférica en dos ojos del grupo control y dos del grupo estudio.

\section{DISCUSIÓN}

Los retratamientos son unas maniobras que se realizan en un porcentaje bajo pero significativo de

Tabla I. Datos preoperatorios comparativos entre grupo control y estudio

\begin{tabular}{|c|c|c|c|}
\hline & Grupo control & Grupo estudio & \\
\hline Edad media & 37 DE 9,5 años & 37,4 DE 8,5 años & $(\mathrm{p}=0,910)$ \\
\hline Sexo & 6 Hombres $(42,8 \%)$ & 7 Hombres $(50 \%)$ & $(\mathrm{p}=0,775)$ \\
\hline Paquimetría & 483,71 DE 31,01 $\mu$ & 457,36 DE $39,93 \mu \mathrm{m}$ & $(\mathrm{p}=0,105)$ \\
\hline K (Curvatura corneal) & 40,79 DE 1,40 dioptrías & 40,38 DE 1,73 dioptrías & $(\mathrm{p}=0,589)$ \\
\hline
\end{tabular}

DE: Desviación estándar 
Tabla II. Grupo control: tratamiento en lecho

\begin{tabular}{rccccccccccc}
\hline Lecho & Edad & Sexo & $\begin{array}{c}\text { E.E. } \\
\text { Pre }\end{array}$ & $\begin{array}{c}\text { A.V. E } \\
\text { Pre. }\end{array}$ & $\begin{array}{c}\text { A.V. C } \\
\text { Pre. }\end{array}$ & $\begin{array}{c}\text { Paquimetri } \\
\text { micras }\end{array}$ & K & $\begin{array}{c}\text { TTO. } \\
\text { Ablación } \mu\end{array}$ & $\begin{array}{c}\text { E.E. } \\
\text { Post. }\end{array}$ & $\begin{array}{c}\text { A.V.E. } \\
\text { Post. }\end{array}$ & $\begin{array}{c}\text { A.V. C. } \\
\text { Post. }\end{array}$ \\
\hline 1 & 37 & H & 1,25 & 0,6 & 1 & 458 & 38,4 & 18 & - & 1 & 1 \\
2 & 51 & M & 1,75 & 0,6 & 0,9 & 448 & 42,3 & 28 & $-0,5$ & 0,8 & 0,9 \\
3 & 33 & H & 1 & 0,5 & 1 & 425 & 41,1 & 12 & - & 1 & 1 \\
4 & 48 & H & 1,5 & 0,4 & 0,8 & 470 & 42,8 & 22,5 & - & 0,9 & 0,9 \\
5 & 44 & M & 1 & 0,8 & 1 & 485 & 39,1 & 14 & - & 1 & 1 \\
6 & 25 & M & 1 & 0,6 & 0,9 & 473 & 40,5 & 12 & $-0,25$ & 0,8 & 0,9 \\
7 & 24 & M & 2 & 0,6 & 1 & 502 & 41 & 25,9 & - & 1 & 1 \\
8 & 42 & M & 3 & 0,3 & 0,7 & 528 & 42,1 & 38 & $-0,5$ & 0,6 & 0,7 \\
9 & 26 & H & 2,0 & 0,4 & 0,8 & 444 & 42,5 & 26 & - & 0,8 & 0,8 \\
10 & 46 & M & 0,75 & 0,8 & 1 & 496 & 38,7 & 16 & - & 0,9 & 0,9 \\
11 & 42 & H & 1,75 & 0,7 & 0,9 & 532 & 42 & 26 & - & 0,9 & 0,9 \\
12 & 36 & M & 2 & 0,6 & 1 & 493 & 39,5 & 28 & - & 1 & 1 \\
13 & 36 & H & 1,5 & 0,7 & 1 & 514 & 40,1 & 20 & - & 1 & 1 \\
14 & 34 & H & 2,25 & 0,6 & 1 & 504 & 41 & 31 & - & 1 & 1 \\
\hline \hline
\end{tabular}

EE: equivalente esférico, AVE pre: agudeza visual espontánea pre-retratamiento, AVC pre: agudeza visual corregida pre-retratamiento, AVE post: a. v. espontánea post-retratameinto, AVC post: agudeza visual corregida post-retratamiento, TTO. ablación: ablación realizada durante el tratamiento expresada en micras, K: curvatura corneal en dioptrías.

Tabla III. Grupo estudio: tratamiento en cara interna del colgajo corneal

\begin{tabular}{|c|c|c|c|c|c|c|c|c|c|c|c|}
\hline Lecho & Edad & Sexo & $\begin{array}{l}\text { E.E. } \\
\text { Pre }\end{array}$ & $\begin{array}{l}\text { A.V. E } \\
\text { Pre. }\end{array}$ & $\begin{array}{l}\text { A.V. C } \\
\text { Pre. }\end{array}$ & $\begin{array}{l}\text { Paquimetri } \\
\text { micras }\end{array}$ & K & $\begin{array}{c}\text { TTO. } \\
\text { Ablación } \mu\end{array}$ & $\begin{array}{l}\text { E.E. } \\
\text { Post. }\end{array}$ & $\begin{array}{l}\text { A.V.E. } \\
\text { Post. }\end{array}$ & $\begin{array}{l}\text { A.V. C. } \\
\text { Post. }\end{array}$ \\
\hline 1 & 45 & M & -1 & 0,7 & 1 & $430 \mu$ & 41,5 & $12 \mu$ & - & 1 & 1 \\
\hline 2 & 23 & M & $-2,75$ & 0,5 & 0,8 & 464 & 38,6 & 33,6 & $-0,5$ & 0,7 & 0,8 \\
\hline 3 & 25 & $\mathrm{H}$ & $-3,25$ & 0,3 & 0,8 & 468 & 39,5 & 51,8 & - & 0,8 & 0,8 \\
\hline 4 & 42 & $\mathrm{M}$ & $-0,6$ & 0,8 & 1 & 415 & 36,8 & 10 & - & 1 & 1 \\
\hline 5 & 29 & M & $-1,6$ & 0,6 & 0,9 & 430 & 40,8 & 26 & - & 0,9 & 0,9 \\
\hline 6 & 47 & $\mathrm{H}$ & $-1,5$ & 0,5 & 1 & 439 & 40,4 & 22 & - & 1 & 1 \\
\hline 7 & 44 & $\mathrm{H}$ & $-1,25$ & 0,4 & 0,8 & 452 & 38,1 & 18 & - & 0,8 & 0,8 \\
\hline 8 & 34 & $\mathrm{H}$ & $-1,25$ & 0,7 & 1 & 445 & 42,5 & 12 & - & 1 & 1 \\
\hline 9 & 49 & M & -1 & 0,5 & 1 & 550 & 41,6 & 14 & - & 0,9 & 1 \\
\hline 10 & 50 & $\mathrm{H}$ & $-1,5$ & 0,4 & 0,7 & 430 & 43,0 & 22 & - & 0,7 & 0,7 \\
\hline 11 & 38 & M & $-1,5$ & 0,6 & 0,9 & 434 & 39,5 & 18,6 & - & 0,9 & 0,9 \\
\hline 12 & 34 & $\mathrm{H}$ & $-1,5$ & 0,7 & 1 & 456 & 40,6 & 16,6 & - & 1 & 1 \\
\hline 13 & 23 & M & $-1,5$ & 0,7 & 1 & 450 & 40,8 & 18,5 & $-0,25$ & 0,9 & 0,9 \\
\hline 14 & 35 & M & -2 & 0,6 & 1 & 540 & 41,7 & 25,9 & - & 1 & 1 \\
\hline
\end{tabular}

EE: equivalente esférico, AVE pre: agudeza visual espontánea pre-retratamiento, AVC pre: agudeza visual corregida pre-retratamiento, AVE post: agudeza visual espontánea post-retratameinto, AVC: agudeza visual corregida post-retratamiento, K: curvatura corneal en dioptrias, TTO. ablación: ablación realizada durante el tratamiento expresada en micras.

cirugías (1-4). Este porcentaje probablemente vaya aumentando debido a la progresiva exigencia de los pacientes. Sin embargo se deben extremar las precauciones ya que en algunos casos debemos retratar córneas ya bastante adelgazadas por tratamientos previos y debemos evitar someter al paciente a un riesgo importante. Debido a estas circunstancias y al desconocimiento que existía sobre los límites exactos del lecho, del colgajo, etc. el porcentaje de ectasias ha ido aumentando $(5,6,10)$. Además se han descrito cambios significativos en la curvatura de la cara posterior de la córnea tras los retrata- mientos (11) en pacientes sin ectasia pero que traducen la importancia de evaluar adecuadamente a los pacientes antes de repetir la cirugía para no caer en las complicaciones.

Uno de los factores más importantes en la patogenia de la ectasia es un lecho estromal reducido (probablemente por debajo de 250 micras), aunque no siempre sucede así ya que con seguridad existen más causas (10).

Ese adelgazamiento excesivo del lecho se produce por una ablación demasiado profunda a su vez condicionada por otros dos factores: porque los 
microqueratomos son todavía muy imprecisos en su profundidad de corte $(12,13)$ y porque en ocasiones las ablaciones son diferentes a las programadas (14). Esta situación se complica en los retratamientos dada la diferencia que hay entre el grosor del colgajo (calculada en el corte del LASIK inicial) con el grosor una vez levantado el colgajo para el retoque probablemente por la hiperplasia epitelial compensadora $(15,16)$.

Maldonado publicó un trabajo describiendo una nueva técnica de retratamiento en ojos que presentaban un estroma posterior insuficiente para la ablación necesaria (7) así como una instrumentación para facilitar la cirugía (17). Una de las razones más poderosas que alegaba para defender su técnica reside en que el estroma existente en el colgajo no contribuye a la integridad tectónica de la córnea tanto como el lecho estromal, por ello hipotetizaba que los retratamientos mediante la técnica de ablación en la cara interna del disco servirían para prevenir futuras ectasias corneales, aconsejando además la conservación de la membrana de Bowman.

Los retratamientos en la cara interna del disco corneal según su técnica son seguros y eficaces y representan una buena solución para no adelgazar excesivamente el lecho estromal residual y así evitar la aparición de ectasias corneales según los resultados aportados por el propio autor (7), el mismo autor ha demostrado mayores cambios en la curvatura de la córnea posterior con la técnica convencional que con su técnica (18).

En este trabajo nosotros hemos analizado en un estudio prospectivo con grupos control y estudio la eficacia y seguridad del tratamiento convencional sobre el lecho residual estromal comparado con el tratamiento realizado en la cara interna del disco. Aunque la técnica es similar a la de Maldonado nuestro concepto del tratamiento es parcialmente diferente. Este autor recomienda no realizar tratamientos que dejen el disco corneal con un grosor inferior a 150 micras y un lecho no inferior a 250 micras mientras que para nosotros los límites son de 100 y 300 micras respectivamente. Nos gusta dejar un disco de 100 micras para dejar un amplio margen sin aproximarnos a la cifra límite de las 250 micras en lecho, ya que factores como ablaciones mayores de las esperadas (14) e hiperplasias del epitelio $(15,16)$ pueden provocar errores de cálculo muy significativos. Además nosotros, tal como describimos en el apartado correspondiente, realizamos en la primera cirugía una paquimetría intrao- peratoria para tener calculado el grosor del colgajo. De esta manera sabemos de manera muy aproximada su valor y a partir de esta medida decidir donde tratar y hacer el cálculo de la ablación.

Los resultados obtenidos por nosotros en los tratamientos de la cara interna son similares a los ya publicados $(7,9)$. Asimismo la eficacia para corregir la refracción residual en los dos grupos que componen nuestro estudio es similar, ya que no existen diferencias significativas en los resultados. Algo parecido sucede con la agudeza visual, puesto que es similar en ambos grupos no habiendo diferencias estadísticamente significativas. Por otra parte no hay pérdida de agudeza visual de dos líneas en ninguno de los ojos tratados con ninguna de las dos técnicas ni se observan alteraciones en el colgajo corneal por lo que puede considerarse que en ambos grupos la técnica quirúrgica es muy segura.

Nuestro estudio presenta varias limitaciones. Dadas las escasas complicaciones que presenta en general la cirugía refractiva no era de esperar que aparecieran de forma significativa en un estudio compuesto por 28 ojos.

Por otra parte los defectos residuales que hemos tratado han sido de pocas dioptrías. Además, aunque los grupos control y estudio eran muy similares en cuanto a sus características, es difícil que sean absolutamente homogéneas especialmente considerando las diferencias interindividuales en las respuestas, por ello en el momento actual estudiamos un grupo de pacientes con retratamiento bilateral donde en un ojo se trata en lecho y en otro en cara interna del colgajo.

En conclusión la técnica descrita por Maldonado de retratamiento en cara interna presenta una eficacia y seguridad similar a la técnica convencional sobre el lecho en la población estudiada.

\section{BIBLIOGRAFÍA}

1. Hu DJ, Feder RS, Basti S, Fung BB, Rademaker AW, Stewart $P$, et al. Predictive formula for calculating the probability of LASIK enhancement. J Cataract Refract Surg 2004; 30: 363-368.

2. Walter KA, Stevenson AW. Effect of environmental factors on myopic LASIK enhancement rates. J Cataract Refract Surg 2004; 30: 798-803.

3. Netto $M V$, Wilson SE. Flap lift for LASIK retreatment in eyes with myopia. Opthalmology 2004; 111: 1362-1367.

4. Perlman EM, Reinert SE. Factors influencing the need for enhancement after laser in situ keratomileusis. J Refract Surg 2004; 20: 783-789. 
5. Amoils SP, Deist MB, Gous P, Amoils PM. Iatrogenic keratectasia after laser in situ keratomileusis for less than -4.0 to -7.0 diopters of myopia. J Cataract Refract Surg 2000; 26: 967-977.

6. Argento C, Consentino MJ, Tytium A, Rapetti G, Zarate J. Corneal ectasia after laser in situ keratomileusis. J Cataract Refract Surg 2001; 27: 1440-1448.

7. Maldonado MJ. Undersurface ablation of the flap for laser in situ keratomileusis retreatment. Ophthalmology 2002; 109: 1453-1464.

8. Pérez-Santonja JJ, Medrano M, Ruiz-Moreno JM, Cardona-Ausina C, Alió JL. Flaprhexis circular: una técnica minuciosa para el retratamiento tras lasik. Arch Soc Esp Oftalmol 2001; 76: 303-308.

9. Versace P, Watson SL. Cornea-sparing laser in situ keratomileusis: ablation on the flap. J Cataract Refract Surg 2005; 31: 88-96.

10. Lifshitz T, Levy J, Klemperer I, Levinger S. Late bilateral keratectasia after LASIK in a low myopic patient. J Refract Surg 2005; 21: 494-496.

11. Rani A, Murthy BR, Sharma N, Fitiyal JS, Vajpayee RB, Pandey RM, et al. Posterior corneal topographic changes after retreatment LASIK. Opthalmology 2002; 109: 1991-1995.

12. Solomon KD, Donnenfeld E, Sandoval HP, Al Sarraf O, Kasper TJ, Holxer MP, et al. Flap thickness accuracy: comparison of 6 microkeratome models. J Cataract Refract Surg 2004; 30: 964-977.

13. Hammer T, Hanschke R, Worner I, Wilhelm FW. Evaluation of four microkeratome models: quality and reproducibility of cut edge and cut surface as determined by scanning electron microscopy. J Refract Surg 2005; 21: 454462.

14. Flanagan GW, Binder PS. The theoretical vs. measured laser resection for laser in situ keratomileusis. J Refract Surg 2005; 21: 18-27.

15. Muallem MS, Yoo SH, Romano AC, Marangon FB, Schiffman JC, Culbertson WW. Flap and stromal bed thickness in laser in situ keratomileusis enhancement. J Cataract Refract Surg 2004; 30: 2295-2302.

16. Randleman JB, Hewitt SM, Lynn MJ, Stulting RD. A comparison of 2 methods for estimating residual stromal bed thickness before repeat LASIK. Ophthalmology 2005; 112: 98-103.

17. Maldonado MJ. Laser-assisted in situ keratomileusis posterior ablation platforum. Arch Ophthalmol 2005; 123. 988-990.

18. Maldonado MJ, Nieto JC, Díez Cuenca M, Piñero DP. Posterior corneal curvature changes after undersurface ablation of the flap and in-the-bed LASIK retreatment. Ophthalmology 2006; 113: 1918-1926. 